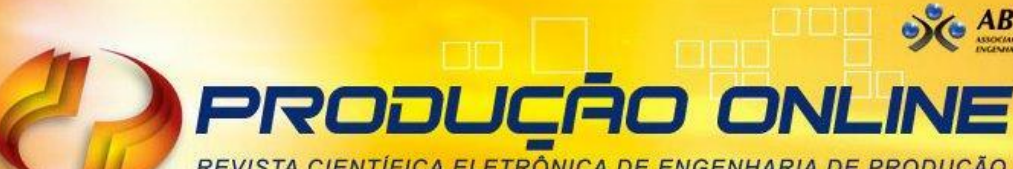 \\ REVISTA CIENTIFICA ELETRO̊NICA DE ENGENHARIA DE PRODUÇÃO \\ ISSN 1676-1901
}

\section{PROPOSTA DE UM MODELO DE PROGRAMAÇÃO LINEAR PARA OTIMIZAÇÃO DO PLANEJAMENTO AGREGADO DE PRODUÇÃO DE BROCAS PARA EMPRESA MULTINACIONAL}

\section{PROPOSAL OF A LINEAR PROGRAMMING MODEL FOR OPTIMIZATION OF THE AGGREGATE PRODUCTION PLANNING FOR MULTINATIONAL COMPANY OF DRILLS}

\author{
Gustavo Gassen*E-mail: gassen86@hotmail.com \\ Odacir Deonisio Graciolli*,** E-mail: odgracio@ucs.br \\ Leonardo Dagnino Chiwiacowsky*, ${ }^{* *}$ E-mail: Idchiwiacowsky@ucs.br \\ Alexandre Mesquita*, ${ }^{* *}$ E-mail: amesquit@ucs.br \\ *Universidade de Caxias do Sul (UCS), Caxias do Sul, RS \\ ${ }^{* *}$ Programa de Pós-Graduação em Engenharia de Produção (PPGEP)
}

\begin{abstract}
Resumo: A complexidade dos processos organizacionais e a necessidade das empresas industriais em aprimorar seus resultados exigem que elas busquem, permanentemente, alternativas eficazes para o planejamento e gerenciamento de sistemas produtivos, que geralmente impactam no desempenho de toda a cadeia de suprimentos. Nesse contexto, a utilização de modelos e ferramentas de otimização pode trazer grandes benefícios para a indústria. Esse trabalho apresenta um modelo de programação linear para a otimização do planejamento agregado da fabricação de itens de uma indústria do setor metalmecânico (conjunto de famílias de brocas). A função objetivo do modelo proposto maximiza as margens de contribuição dos produtos, respeitando as restrições de capacidade produtiva e atendimento à demanda para um planejamento multiperíodos. Por meio do modelo matemático, são avaliados diferentes cenários que podem ser úteis no gerenciamento das atividades de planejamento da produção. A metodologia aplicada mostrou-se útil para a otimização do planejamento agregado da produção, auxiliando na definição do mix de produtos a serem fabricados, bem como os resultados para diferentes cenários, subsidiando a tomada de decisões gerenciais. Para o cenário que considera as condições do plano de produção realizado atualmente pela empresa, o modelo proposto apresentou uma melhoria na margem de contribuição de mais de 7\%. Além de auxiliar na tomada de decisões, o emprego do modelo matemático proporciona a geração de uma base de dados importante para os gerentes, que poderão reportar aos executivos as características do planejamento de produção e resultados da companhia, de forma rápida, direta e quantitativa.
\end{abstract}

Palavras-chave: Planejamento Agregado. Programação Linear. Planejamento da Produção.

Abstract: The complexity of the organizational processes and the needs of improving their results make the industrial companies to search for effective alternatives for the planning and management of production systems, which generally impact in the performance of the entire supply chain. In this context, the use of optimization models and tools can give the organization great benefits. The present study proposes a linear programming model for the optimization of the aggregate planning for the manufacturing of items of a metalworking sector company (drill bits company). The objective function of the proposed model maximizes the contribution margins of the products, respecting the capacity and demand constraints for multiperiod planning. This study also presents evaluation scenarios that may be useful in the management of the manufacturing activities. The applied methodology shown itself to be

Revista Produção Online. Florianópolis, SC, v. 19, n. 1, p. 21-43, 2019. 
powerful to define and improve the aggregate planning activities, helping to determine the products mix and upgrading the management decisions, which confirms the viability of the applied method and reaches the goals. Assuming a specific scenario representing the current conditions of the production plan conducted in the company, the proposed model achieved an improvement in the contribution margin of more than $7 \%$. In addition to assisting in decision making, the use of the mathematical model allows the generation of an important database for managers, who can report to the executives the characteristics of the production planning and company's results, in a quick, direct and quantitative way.

Keywords: Aggregate Planning. Linear Programming. Production Planning.

\section{INTRODUÇÃO}

Diante da competitividade empresarial contemporânea, na busca pela melhoria de desempenho das empresas através de estratégias gerenciais de produção, o uso de técnicas e ferramentas de pesquisa operacional permite otimização de recursos e redução de custos e, consequentemente, melhor entendimento, análise e solução de problemas de decisão (ARENALES et al., 2015). De uma forma geral, a pesquisa operacional contempla um conjunto de conceitos matemáticos, estatísticos e técnicas de inteligência artificial utilizado para modelar problemas com o objetivo de gerar soluções otimizadas. Em âmbito nacional, a pesquisa operacional existe desde a década de 1970, mas somente agora vem conquistando as empresas (BARCELOS, EVANGELISTA, SEGATTO, 2012). Provavelmente, isto se deve ao fato de que até os anos de 1990, a implantação de algumas ferramentas de pesquisa operacional era de difícil realização, devido ao desenvolvimento tecnológico de softwares e da capacidade de processamento dos computadores na época (ANDRADE, 2015).

Krajewski, Ritzman e Malhotra (2009) entendem que o cenário competitivo em que as empresas se encontram, atualmente, gera certa tensão por parte das organizações que não possuem um gerenciamento sólido e não estão focadas no atendimento ao cliente. Para melhorar a sua competitividade, elas necessitam aprimorar as suas estratégias competitivas, tornando seus processos mais eficazes e formando uma cadeia de valor que se estenda a seus clientes e fornecedores.

Algumas empresas ainda são dependentes da experiência e da percepção de seus gestores para as tomadas de decisão organizacionais (OLIVEIRA, SILVEIRA, FERREIRA, 2010). Na visão de Andrade (2015), as decisões gerenciais possivelmente estejam corretas quando envolvem situações rotineiras, entretanto, 
podem não ter sucesso devido a limitações de cunho pessoal do administrador. Além disso, cada vez mais o mercado exige mudanças nas organizações, de forma que a equipe gerencial é obrigada a tomar decisões rápidas, sendo pressionada por resultados eficazes para a empresa, pois, caso contrário, essas decisões podem levar a índices de desempenho insuficientes.

Slack et al. (1997) afirmam que o Planejamento e Controle da Produção (PCP) tem o propósito de garantir que a produção ocorra eficazmente e que a empresa consiga fabricar seus produtos conforme necessário, fazendo com que os recursos produtivos estejam disponíveis na quantidade adequada, no momento adequado e com qualidade. Atualmente, há diferentes modelos e ferramentas para subsidiar a tomada de decisão em contextos evolvendo sistemas produtivos (RODRIGUES, COSTA, CARMO, 2013), bem como métodos para estimar escolhas ótimas (ONWUBOLU, 2002).

Por sua vez, a Pesquisa Operacional (PO) apresenta-se como uma área que reúne diferentes técnicas e ferramentas para o desenvolvimento de abordagens de solução para problemas de tomada de decisão. Dependendo da forma adotada para modelagem do problema de interesse, isto é, linear ou não-linear, a PO possibilita o desenvolvimento de uma variedade de métodos científicos visando à determinação do melhor caminho de projeto ou operação, geralmente sob regime de recursos escassos (Arenales et al., 2015).

Especificamente, a Programação Linear (PL) é uma das ferramentas mais difundidas da PO. Trata-se de um conjunto de técnicas matemáticas que tem por objetivo otimizar a solução de problemas cuja modelagem é caracterizada por equações e/ou inequações lineares. Silva Filho, Cezarino e Ratto (2009) propuseram um modelo matemático baseado em programação linear para geração de planos agregados que podem ser utilizados pelos gestores na elaboração de cenários de produção, por meio do uso de um aplicativo baseado no suplemento Solver da planilha Excel. Trabalhos mais recentes (ROCHA et al., 2012; SILVEIRA et al., 2013) apresentam a utilização da programação linear em aplicações de otimização da programação da produção e de redução de custos. Ambos os estudos identificaram 
melhorias nos processos produtivos após a aplicação da ferramenta, respeitando as restrições do sistema.

Outro fator importante para o gestor na administração da manufatura é a possibilidade de simular novos cenários de forma rápida, o que pode ser viabilizado pelo emprego de um modelo de PL, a fim de identificar as limitações do sistema, ou mesmo exibir ganhos que poderiam ser obtidos por meio de investimentos na ampliação da capacidade produtiva. Estes resultados exibidos com uso do modelo matemático adequado proporcionam aos gestores um maior conhecimento das características produtivas, e dão maior confiabilidade a suas decisões.

Por meio da apresentação dos resultados financeiros dos diversos cenários avaliados e da montagem de uma base de dados, o modelo de PO se torna uma importante ferramenta para os gerentes reportarem os resultados da companhia aos executivos, que necessitam compreender de forma rápida, direta e quantitativa as características do planejamento de produção.

Desta forma, o presente trabalho tem como objetivo propor um modelo de programação linear para a otimização de um problema de Planejamento Agregado da Produção (PAP), em uma empresa multinacional, fabricante de brocas, localizada no Estado do Rio Grande do Sul. Mais precisamente, o estudo teve como base a produção de uma família específica de brocas, para um período de planejamento da produção de seis meses. Todos os dados necessários para o desenvolvimento do modelo proposto foram obtidos do sistema ERP SAP ${ }^{\circ}$ da empresa, por meio de acesso aos relatórios setoriais. Assim, foi efetuada uma coleta ampla e rigorosa, buscando-se o maior número de informações possível sobre o processo em estudo, aliada a uma análise criteriosa dos dados obtidos, combinada com a realização de ajustes necessários para que fossem gerados resultados os mais próximos possíveis da realidade vivenciada pela empresa.

Os valores de estoque inicial de cada produto foram obtidos do sistema ERP SAP $\AA$, conforme descrito anteriormente, tendo sido coletada a quantidade em estoque no mês anterior ao período de planejamento utilizado no estudo. A demanda prevista para os meses de planejamento foi determinada pelo processo de S\&OP (Sales and Operation Planning) da empresa, que emprega ferramentas estatísticas 
para a previsão de vendas, levando em consideração histórico de vendas, projeções, crescimento do mercado, dentre outras medidas de relevância.

O trabalho está estruturado de forma a apresentar, em sequência, o referencial teórico, com os principais conceitos e exemplos de aplicação ligados ao modelo de programação linear para o planejamento agregado da produção, de forma a definir o mix de produtos a serem produzidos. Prosseguindo, são descritos os procedimentos metodológicos, com os modelos, critérios e plataformas computacionais utilizados, além das características da empresa onde o trabalho foi aplicado; seguidos pela análise e discussão dos resultados; e, por fim, a conclusão.

\section{REFERENCIAL TÓRICO}

Nas estratégias de planejamento e gestão de operações, de logística e da cadeia de suprimentos, é necessário tratar com um conjunto de atividades funcionais (produção, controle de estoques, transportes, etc.) que exige o tratamento de cenários cada vez mais complexos. Comumente, estes cenários envolvem muitas variáveis, notadamente ao longo da cadeia de suprimentos, pela qual matérias-primas vão sendo convertidas em produtos acabados (BALLOU, 2006).

De acordo com Lachtermacher (2002), todo tipo de empresa possui escassez de algum produto ou matéria-prima, originando, assim, desafios para o gerenciamento de seus recursos de forma eficiente e eficaz, com o objetivo de maximizar ou minimizar variáveis como lucro, custo, receita, número de produtos, dentre outras. Processos de otimização de recursos podem ser aplicados em diversas áreas como, por exemplo, na determinação de mix de produtos (CASSEL, VACCARO, 2007), no escalonamento da produção (CAVALCANTE, 1998), no roteamento e logística (AZUMA, 2011), no planejamento financeiro (DOS REIS, MARTINS, 2001) e na análise de projetos (TANCREDI, 2009).

Na visão de O'Keefe (2004 apud DONATO, 2008), o S\&OP é apontado como uma ferramenta utilizada pela alta gerência para elaborar o planejamento estratégico do negócio e executar as ações necessárias, como gerenciar, monitorar e controlar os cenários de vendas, produção e estoques, bem com avaliar as definições de risco e a variabilidade dos planos de negócio. Corrêa et al. (1997 apud OLIVEIRA, 2011) e 
Wallace (2004 apud TOMAS, SATO, ALCANTARA, 2012) permitem caracterizar o S\&OP como um mecanismo que reintegra os diversos níveis desagregados de processos visando às tomadas de decisão, com o foco em solucionar os problemas de mix de produtos mais rapidamente.

De acordo com Vollmann, Berry e Whybark (1997), o Planejamento e Controle da Produção é parte de um sistema de informação do sistema produtivo, com ênfase em materiais, máquinas, mão de obra e fornecedores. A eficiência deste sistema pode fazer com que a empresa tenha vantagens competitivas no mercado em que está inserida, porém essa eficiência de hoje pode não ser a de amanhã, pois mudanças ocorrem a todo o momento e o sistema deve estar preparado para responder a estas mudanças, cumprindo com os resultados desejados.

O horizonte de planejamento do sistema produtivo pode ser dividido em três níveis, o longo, o médio e o curto prazo, mas não existe um padrão quanto ao tempo de abrangência de cada período (TUBINO, 2000). Normalmente, empresas que possuem uma baixa flexibilidade de resposta às variações de demanda terão horizontes de planejamento mais longos. Por estas empresas tomarem as decisões com mais antecedência, maior será a probabilidade de ocorrerem erros (TUBINO, 2000).

Para Moreira (1996), as análises de demanda e mercado realizadas com base no longo prazo fazem com que as decisões sobre a capacidade possam ser tomadas no futuro. Essas decisões de capacidade influenciam diretamente no planejamento das instalações da empresa, e consecutivamente, influenciarão na contratação de mão de obra e aquisição de equipamentos.

Segundo Slack et al. (1997), para o planejamento e controle de longo prazo, os gestores estão mais focados em planejar, e não controlar. Com base neste horizonte de tempo, é analisado o que se pretende fazer, quais os recursos que serão necessários e quais os objetivos que se deseja alcançar.

O planejamento de médio prazo, também conhecido como Planejamento Agregado da Produção (PAP) (CHERAGHALIKHANI, KHOSHALHAN, MOKHTARI, 2019), é definido como o estágio intermediário entre o planejamento da capacidade e da produção, ou seja, do planejamento de longo e de curto prazo, respectivamente. A 
longo prazo, é fixada a capacidade produtiva da empresa e, a curto prazo, a produção é determinada de acordo com as limitações dos estágios anteriores (MOREIRA, 1996).

Segundo Slack et al, (1997), o planejamento e controle de médio prazo é o planejamento realizado com mais detalhes, sendo feito também o replanejamento, caso necessário. Nesse horizonte de análise, é realizada uma estimativa da demanda global que a operação deve suprir de uma forma parcialmente desagregada (NAM, LOGENDRAN, 1992).

O planejamento de médio prazo tem como objetivo atender às irregularidades da demanda do mercado por meio da utilização dos recursos da empresa. Como nem sempre a empresa tem condições de atender à demanda, os responsáveis pelo planejamento têm como tarefa encontrar uma opção de compromisso capaz de considerar a variabilidade da demanda e a disponibilidade da capacidade produtiva (MONKS, 1987).

Slack et al. (1997) apontam que durante o planejamento de médio prazo, diferentes categoriais deverão ser identificadas e as quantidades de cada uma precisarão ser definidas. Da mesma forma, neste momento é necessário pensar no desenvolvimento dos planos contingenciais, para que o planejamento e controle tornem-se mais simples de serem realizados em um horizonte de curto prazo, caso necessário. Segundo os mesmos autores, o planejamento e controle de curto prazo assumem a demanda avaliada de forma totalmente desagregada e, embora muitos dos recursos já tenham sido definidos, não se descartam realocações caso ocorram alterações em relação ao plano previamente elaborado. Essas mudanças no curto prazo, realizadas pela gestão, provavelmente não serão embasadas em cálculos detalhados com relação aos seus impactos, mas sim em uma compreensão geral das prioridades que auxiliará sua tomada de decisão.

\subsection{Planejamento Agregado da Produção}

O Planejamento Agregado da Produção (PAP) normalmente é realizado no 
horizonte de médio prazo, entre 3 meses a 1 ano, e consiste no processo de planejar o ritmo de produção, a disponibilidade de mão de obra, os estoques e outras variáveis controláveis (MONKS, 1987). Também conhecido como Plano de Produção (PP Production Planning), o PAP provavelmente é um dos módulos menos conhecidos pelos responsáveis pelo PCP, porém, se ele for gerado de uma forma bem elaborada pela empresa, pode trazer resultados favoráveis. A possível qualidade dos resultados alcançados fundamenta-se no fato de que esse módulo conecta os objetivos estratégicos com a produção, sincronizados através dos objetivos de vendas, recursos disponíveis e o orçamento financeiro da empresa (VOLLMANN, BERRY, WHYBARK, 1997).

De acordo com Monks (1987) e Moreira (1996), o planejamento agregado conecta as atividades de planejamento de longo e curto prazo. Considera-se "agregado" pois ele não se concentra sobre bens e serviços individuais, mas os agrupa em famílias ou pseudo-produtos, como clientes atendidos, número de motores ou toneladas de metais. Diante da variedade de produtos fabricados pela empresa, tornase inviável o planejamento de toda a produção com a demanda prevista individualmente, daí o termo "agregado", que tem o objetivo de considerar medidas unificadas para os produtos, ou no máximo para alguma linha de produto. Tomando como base um período de tempo futuro relativamente curto (inferior a um ano), o planejamento agregado deve determinar como e o quanto deverá ser produzido para atender à demanda para o período considerado. Esse plano envolverá diversas alternativas e, para cada uma delas, haverá diferentes custos de produção associados.

Segundo Vollmann, Berry e Whybark (1997), o PAP possui uma ligação direta tanto com a manufatura, quanto com o gerenciamento de alto nível da empresa, o qual dita as estratégias do negócio.

É importante ressaltar a relação do PAP com as diversas áreas funcionais da empresa, e apontar que elas estão diretamente ligadas aos objetivos do planejamento agregado. Destacam-se seis objetivos principais para o planejamento agregado da produção, segundo Krajewski e Ritzman (2001 apud LARA, 2003): minimização de custos ou maximização dos lucros, maximização do nível de serviço do cliente, 
minimização dos investimentos em inventário, minimização das mudanças nas taxas de produção, minimização nas mudanças no nível de força de trabalho, e, por fim, maximização na utilização de máquinas e equipamentos.

Tubino (2000) identifica várias técnicas existentes que podem ser utilizadas para a elaboração do PAP e que, normalmente, são divididas em modelos matemáticos e modelos empíricos e semi-empíricos, baseados em estratégias de tentativa e erro. A primeira opção está focada em soluções otimizadas, já a segunda se baseia na experiência e no bom senso dos planejadores.

$\mathrm{Na}$ abordagem matemática, Corrar e Theóphilo (2004) apontam que a Programação Linear (PL) é uma das ferramentas mais importantes da Pesquisa Operacional (PO), sendo normalmente utilizada para a solução de problemas que visam a atingir determinado objetivo de otimização, melhorando o emprego dos recursos disponíveis. Existem diversos tipos de problemas que podem ser modelados através da PL, entre eles, as decisões para definir o mix de produção. Para Chopra e Meindl (2004), o emprego da programação linear no planejamento agregado tem a função de maximizar lucros, atendendo à determinada demanda, e segundo os autores, ela é uma ferramenta muito eficaz e pode ser utilizada pelas empresas para esse objetivo.

Moreira (1996) cita o modelo de programação linear como um método para obter a solução do planejamento agregado. Devido à variação da demanda, em alguns meses, os recursos da empresa podem estar ociosos, enquanto que, em outros meses, podem estar sobrecarregados. Portanto, a tarefa de balancear o uso dos recursos produtivos durante todo o período, normalmente, é o mais comum dos problemas de PAP que podem ser solucionados pela ferramenta matemática.

Moreira (1996) e Vollmann, Berry e Whybark (1997) sugerem um modelo específico de programação linear para resolver o problema do PAP, o qual, segundo eles, deve ser considerado como uma formulação possível, já que existe certa flexibilidade devido às variáveis de cada problema. Os autores ainda enfatizam que o modelo de programação linear apresentado não pretende ser definitivo, pois sempre existe a opção de serem incorporadas outras variáveis e restrições, de acordo com a necessidade e as condições estipuladas pela gerência. 
Desta forma, este trabalho aborda o desenvolvimento e aplicação de um modelo de programação linear para o planejamento agregado da produção, de forma a definir o mix de produtos agregados a serem produzidos, maximizando as margens de contribuição, respeitando as restrições de capacidade produtiva, os níveis de estoque, bem como o atendimento à demanda para um horizonte de $n$ períodos de planejamento. O modelo define a quantidade a ser produzida de produtos agregados para atender diretamente à demanda e/ou destinado ao estoque para atender demandas futuras.

\section{MATERIAIS E MÉTODO}

O modelo de programação linear para a otimização do planejamento agregado da produção foi desenvolvido por meio da definição das variáveis de decisão, da função objetivo e do conjunto de restrições, para o caso de uma empresa multinacional fabricante de ferramentas e localizada no Estado do Rio Grande do Sul. Mais especificamente, o estudo teve como base a produção de um conjunto de 43 famílias (produtos agregados por similaridades) de brocas produzidas pela empresa, para um horizonte de planejamento da produção de 6 meses e períodos definidos em meses. A empresa efetua a agregação das brocas em famílias considerando a similaridade nas características dos produtos e nos processos produtivos. Para cada família, é atribuído um código de identificação e levantadas as informações sobre os processos produtivos das respectivas famílias: fluxos, tempos, custos de produção e margem de contribuição.

As famílias, produtos agregados, são referenciadas neste trabalho simplesmente como produtos e os parâmetros associados a eles são definidos considerando todos os produtos que compõem a família.

As quantidades de estoque inicial de cada produto foram obtidas do sistema de gestão empresarial $S A P^{\circledR}$, que fornece a quantidade em estoque no mês anterior ao primeiro período (mês) de planejamento utilizado no estudo. A demanda prevista para os meses de planejamento é determinada pelo processo de planejamento de vendas, $S \& O P$ da empresa, que utiliza ferramentas estatísticas para a previsão de demandas, 
levando em consideração histórico de vendas, projeções, crescimento do mercado, dentre outras medidas.

A capacidade de produção da empresa está diretamente ligada à disponibilidade de horas dos recursos de produção, representados basicamente por máquinas. As operações para fabricação de brocas são automatizadas e, embora existam operadores trabalhando em um ou mais recursos de produção, suas atividades estão relacionadas ao abastecimento, calibração e manutenção das máquinas, bem como inspeção de qualidade de insumos e produtos.

Dessa forma, considerou-se para este estudo que o aumento da capacidade produtiva de cada recurso, visando ao atendimento da demanda, está ligado à aquisição de novas máquinas. Portanto, contratações e demissões de operadores não são consideradas no modelo.

Também foram levantados os parâmetros referentes às capacidades de produção: quantidades de recursos produtivos, turnos de trabalhos de cada recurso, quantidades e custos de horas regulares e horas extras, horizonte de planejamento dividido em períodos, quantidades de estoques iniciais de cada família, custos referentes à manutenção de estoques e demanda para cada família em cada período de planejamento.

\subsection{Modelo de Otimização}

As variáveis de decisão do modelo de otimização proposto estão relacionadas às quantidades de unidades de cada família de produtos produzidas utilizando horas regulares, horas extras e a produção para estoque. Para facilitar a representação matemática, foi empregada a notação $p$ (produto) para representar uma família de brocas, ou seja, o conjunto de produtos agregados. Portanto, as variáveis de decisão são assim definidas:

$P_{p, t}=$ quantidade de unidades produzidas do produto $p$, no período $t$, utilizando horas regulares;

$P H E_{p, t}=$ quantidade de unidades produzidas do produto $p$, no período $t$, utilizando horas extras;

Revista Produção Online. Florianópolis, SC, v. 19, n. 1, p. 21-43, 2019. 
$E_{p, t}=$ quantidade de unidades em estoque do produto $p$, no período $t$.

A Equação (1) representa a quantidade total de horas disponíveis (Disp $\left.p_{r, t}\right)$ para a produção considerando somente as horas regulares do recurso $r$ para o período $t$, entendendo como recurso produtivo as máquinas que executam os processos de produção.

Disp $_{r, t}=$ NumH $_{r, t} \times$ DiasTrab $_{t} \times E f_{r}$.

A medida $\operatorname{Disp}_{r, t}$ é determinada pela multiplicação do número de horas disponíveis por dia do recurso $r$ em um período $t\left(N u m H_{r, t}\right)$ pelo número de dias trabalhados no período $t$ (DiasTrab ${ }_{t}$ ) e pela eficiência do recurso $r\left(E f_{r}\right)$. Para esse estudo, foi utilizado um padrão de eficiência de $85 \%$ para todos os recursos. Portanto, considerou-se que $15 \%$ da disponibilidade total de horas de produção dos recursos não fica disponível para produzir, ou seja, é um tempo correspondente a paradas de produção programadas, geralmente destinado para manutenções, ou para modelar a ocorrência de paradas não programadas.

A quantidade total de horas disponíveis para a produção, mantendo-se os mesmos recursos, pode ser aumentada com a contratação de horas extras, novos funcionários ou ampliação de turnos. Outra forma de aumentar a disponibilidade total é por meio da aquisição de novos recursos. A disponibilidade total de horas de produção utilizando horas extras é expressa por:

$D i s p H E_{r, t}=N u m H E_{r, t} \times$ DiasTrabHE $E_{t} \times E f_{r}$,

onde $N u m H E_{r, t}$ representa número de horas extras diárias permitidas para o recurso $r$ no período $t$, e DiasTrabHE $E_{t}$ representa a quantidade de dias trabalhados utilizando horas extras no período $t$.

Foi utilizado o conceito de margem de contribuição para definir a função objetivo do modelo proposto. A margem de contribuição de cada produto $p$, representado por $M C_{p}$ na Equação (3), é definida pela diferença entre o preço de venda do produto $p\left(P V_{p}\right)$ e o custo de produção do mesmo $\left(C_{p}\right)$. 
$M C_{p}=P V_{p}-C_{p}$

Da mesma forma, porém, com base no custo de produção utilizando hora extra $\left(C H E_{p}\right)$, a Equação (4) define a margem de contribuição do produto $p$ quando produzido em hora extra $\left(M C H E_{p}\right)$ :

$M C H E_{p}=P V_{p}-C_{1}$.

A função objetivo busca maximizar a margem de contribuição total para atender à demanda dos produtos nos períodos do horizonte de planejamento, bem como minimizar o custo de estocagem. A Equação (5) apresenta a função objetivo do modelo:

$$
F O=\operatorname{Max} \sum_{p=1}^{N p} \sum_{t=1}^{N t}\left(P_{p, t} M C_{p}+P H E_{p, t} M C H E_{p}-E_{p, t} C E_{p}\right)
$$

onde:

$N p=$ número de produtos;

$N t$ = número de períodos do horizonte de planejamento;

$C E_{p}=$ custo de manter em estoque o produto $p$ durante um período.

As restrições do problema estão relacionadas às necessidades de atendimento das demandas de cada produto para cada período e também às limitações definidas pelas disponibilidades de horas para produção dos produtos. A Equação (6a) representa o balanceamento entre a quantidade produzida do produto $p$ num período $t$ (utilizando horas regulares e horas extras) somado ao disponível em estoque do produto $p$ no final do período $t-1$, e a quantidade a ser atendida da demanda do produto $p$ para o período $t\left(D_{p, t}\right)$, assumindo-se que o excedente deve ser estocado:

$P_{p, t}+P H E_{p, t}+E_{p, t-1}=D_{p, t}+E_{p, t}, \quad p=1, \ldots, N p ; t=1, \ldots, N t$

ou

Revista Produção Online. Florianópolis, SC, v. 19, n. 1, p. 21-43, 2019. 
$D_{p, t}=P_{p, t}+E_{p, t-1}-E_{p, t}, \quad p=1, \ldots, N p ; t=1, \ldots, N t$

Para $t=1$, a notação $E_{p, t-1}$ representa o estoque inicial do produto $p\left(E I_{p}\right)$.

O tempo dedicado para produção de produtos com uso do recurso $r$ num período $t$ não deve ultrapassar a disponibilidade de horas de produção regular do recurso $r$ no período $t$, como expresso na Equação (7). Da mesma forma, o tempo dedicado para produção de cada produto com uso do recurso $r$ num período $t$, utilizando horas extras, não deve ultrapassar a disponibilidade de horas de produção com horas extras do recurso $r$ no período $t$, conforme a Equação (8).

$$
\begin{aligned}
& \sum_{p=1}^{N p}\left(P_{p, t} T_{p}\right) \leq \text { Disp }_{r, t}, \quad r=1, \ldots, N r ; t=1, \ldots, N t \\
& \sum_{p=1}^{N p}\left(P H E_{p, t} T_{p}\right) \leq \operatorname{DispHE}_{r, t}, \quad r=1, \ldots, N r ; t=1, \ldots, N t
\end{aligned}
$$

onde:

$N r$ = número de recursos;

$T_{p}=$ tempo em horas para produzir o produto $p$.

Para finalizar o modelo, são incluídas as restrições de não negatividade para as variáveis, conforme definido na Equação (9).

$$
P_{p, t} ; P H E_{p, t} ; E_{p, t} \geq 0, \quad p=1, \ldots, N p ; t=1, \ldots, N t
$$

A capacidade de produção do recurso, que neste trabalho está restrita à disponibilidade em horas do recurso $r$ no período $t$ (Disp $p_{r, t}$ ), foi determinada considerando o uso compartilhado do recurso para produção de produtos que não estão considerados no estudo.

As definições apresentadas para a capacidade de produção do recurso utilizando horas regulares são similares à capacidade de produção utilizando horas 
extras $\left(D i s p H E_{r, t}\right)$. A disponibilidade de horas extras diárias do recurso será considerada somente quando o recurso em questão não apresentar disponibilidade total nos três turnos de trabalho. Caso o emprego de uma determinada máquina já esteja ocorrendo nos três turnos, a disponibilidade de horas extras é considerada somente nos feriados e finais de semana. A soma das disponibilidades de horas de produção em regime normal com as horas extras resulta na capacidade máxima de produção para cada recurso. O custo de produção utilizando horas extras é maior quando comparado ao custo utilizando horas regulares. No entanto, podem ser utilizadas horas extras quando a capacidade de produção em horas regulares somada aos níveis de estoques não forem suficientes para atender a demanda.

Os parâmetros do modelo, como por exemplo, o tempo de produção de cada produto (que representa uma família de produtos agregados), são obtidos pela média dos produtos agregados.

Os parâmetros financeiros utilizados no modelo são: $P V_{p}$ preço de venda unitário do produto $p, C E_{p}$ custo de armazenagem do produto $p$ para o período $t, C_{p}$ custo de produção do produto $p$ utilizando horas regulares e $C H E_{p}$ custo de produção do produto $p$ utilizando horas extras.

\section{RESULTADOS E ANÁLISE}

Para fins de análise gerencial e discussões sobre a potencialidade que os modelos de pesquisa operacional proporcionam, foram avaliados três cenários. $A$ partir do modelo básico, foram feitas as alterações necessárias para representar cada cenário avaliado.

As versões do modelo de programação linear para definir o mix de produção ideal nos cenários avaliados, equações (5) a (9), foram executadas no software de otimização Xpress-MP versão 8 em um computador com 1,3 GHz Intel Core i5 e 4 GB RAM. O tempo de resposta foi de menos de 1 segundo para todos os cenários gerados.

A Tabela 1 relaciona o plano de produção realizado assim como os três cenários avaliados. Na coluna "Demanda" o símbolo "=" indica que o modelo prevê o atendimento exato da demanda prevista, a prescrição “ $\pm 10 \%$ " indica uma relaxação 
na restrição de atendimento à demanda prevista, podendo variar entre 10\% a menos e 10\% a mais; e, por fim, no caso da demanda "Livre", o modelo não precisa atender à demanda prevista. A coluna "Horas Extras" representa a permissão ou não do uso de horas extras. Na coluna "FO", estão representados os valores da função objetivo (margem de contribuição total) de cada cenário. Finalmente, "Melhoria (\%)" representa o percentual de melhora em relação ao realizado (cenário Real).

Tabela 1 - Configuração dos cenários avaliados

\begin{tabular}{ccccc}
\hline Cenário & Demanda & Horas Extras & FO (R\$) & Melhoria (\%) \\
\hline Real & $=$ & Sim & 186.793 & \\
1 & $=$ & Sim & 200.011 & 7,07 \\
2 & $\pm 10 \%$ & Sim & 215.382 & 15,30 \\
3 & Livre & Sim & 409.840 & 119,40 \\
\hline
\end{tabular}

Fonte: Elaborada pelos autores

Apesar do modelo de programação linear permitir o uso de horas extras, o emprego da ferramenta de otimização permitiu a obtenção de soluções indicando o uso de apenas de horas regulares, isto é, sem a necessidade de uso de horas extras em nenhum dos cenários propostos. Isso ocorre em função da capacidade produtiva normal atender à demanda prevista (para os parâmetros do estudo de caso, antecipar a produção em alguns períodos de forma a manter estoque é mais econômico que usar horas extras). No entanto, para casos hipotéticos em que a demanda real observada seja superior ao patamar de $10 \%$ da demanda inicialmente prevista, poderia ser necessário o uso de horas extras para atendê-la, devendo ser salientado que para a avaliação desta nova situação não é exigida qualquer alteração no modelo aqui proposto.

Considerando o cenário real, isto é, a política de produção adotada pela empresa em estudo, a margem de contribuição total do planejamento agregado da produção realizado é de $\mathrm{R} \$ 186.793,00$. Observa-se que neste planejamento proposto pela empresa foram utilizadas horas extras para atender à demanda em alguns períodos. Em outros períodos, houve produção para estoque.

No Cenário 1, o modelo apresentou a margem de contribuição total (FO na Tabela 1) de $R \$ 200.011,00$, que representa um ganho de mais de $7 \%$ em relação ao plano de produção realizado. 
A Tabela 2 apresenta o percentual de ocupação de cada recurso em cada um dos períodos do horizonte de planejamento para o Cenário 1.

Tabela 2 - Ocupação dos recursos para o Cenário 1 (\%)

\begin{tabular}{|c|c|c|c|c|c|c|c|c|c|c|c|c|c|c|c|c|c|c|c|c|c|c|}
\hline \multirow[b]{2}{*}{ Mês } & \multicolumn{22}{|c|}{ Recursos } \\
\hline & 1 & 2 & 3 & 4 & 5 & 6 & 7 & 8 & 9 & 10 & 11 & 12 & 13 & 14 & 15 & 16 & 17 & 18 & 19 & 20 & 21 & 22 \\
\hline 1 & 14 & 60 & 2 & 0 & 12 & 4 & 30 & 3 & 18 & 35 & 11 & 10 & 43 & 1 & 25 & 2 & 11 & 8 & 33 & 100 & 30 & 27 \\
\hline 2 & 33 & 100 & 49 & 0 & 51 & 46 & 24 & 10 & 30 & 68 & 37 & 34 & 87 & 72 & 89 & 38 & 27 & 37 & 45 & 7 & 9 & 60 \\
\hline 3 & 37 & 100 & 99 & 11 & 28 & 43 & 28 & 10 & 10 & 100 & 71 & 32 & 100 & 38 & 100 & 26 & 30 & 46 & 56 & 100 & 50 & 73 \\
\hline 4 & 18 & 100 & 61 & 40 & 27 & 19 & 26 & 6 & 13 & 90 & 53 & 23 & 100 & 17 & 100 & 21 & 42 & 46 & 49 & 100 & 23 & 95 \\
\hline 5 & 100 & 100 & 100 & 18 & 33 & 36 & 19 & 8 & 26 & 53 & 61 & 35 & 78 & 77 & 100 & 40 & 38 & 28 & 43 & 0 & 0 & 75 \\
\hline 6 & 44 & 72 & 100 & 9 & 23 & 46 & 23 & 9 & 11 & 83 & 84 & 40 & 100 & 23 & 100 & 24 & 34 & 55 & 98 & 0 & 0 & 83 \\
\hline
\end{tabular}

Fonte: Elaborada pelos autores

É possível observar que os recursos 2 e 15, por exemplo, têm uma ocupação de $100 \%$ na maioria dos períodos. Estes recursos são gargalos que podem ser avaliados como candidatos a terem sua capacidade expandida. Por outro lado, é possível observar grande ociosidade do recurso 8 que tem uma ocupação máxima de 10\%. Cabe observar que este é o cenário que considera as condições do plano de produção realizado atualmente pela empresa.

Para o Cenário 2, em que é aceita uma violação na restrição de atendimento exato à demanda, podendo variar entre $10 \%$ a menos e $10 \%$ a mais, o modelo apresentou a margem de contribuição total de $R \$ 215.382,00$, o que representa um ganho de mais de $15 \%$ em relação ao plano de produção realizado.

A Tabela 3 apresenta o percentual de ocupação de cada recurso em cada um dos períodos do horizonte de planejamento para o Cenário 2. 
Tabela 3 - Ocupação dos recursos para o Cenário 2 (\%)

\begin{tabular}{crrrrrrrrrrrrrrrrrrrrrrr}
\hline Mês & $\mathbf{1}$ & $\mathbf{1}$ & $\mathbf{2}$ & $\mathbf{3}$ & $\mathbf{4}$ & $\mathbf{5}$ & $\mathbf{6}$ & $\mathbf{7}$ & $\mathbf{8}$ & $\mathbf{9}$ & $\mathbf{1 0}$ & $\mathbf{1 1}$ & $\mathbf{1 2}$ & $\mathbf{1 3}$ & $\mathbf{1 4}$ & $\mathbf{1 5}$ & $\mathbf{1 6}$ & $\mathbf{1 7}$ & $\mathbf{1 8}$ & $\mathbf{1 9}$ & $\mathbf{2 0}$ & $\mathbf{2 1}$ & $\mathbf{2 2}$ \\
\hline 1 & 16 & 91 & 2 & 0 & 30 & 11 & 35 & 5 & 21 & 80 & 8 & 15 & 76 & 13 & 45 & 22 & 16 & 16 & 50 & 100 & 23 & 43 \\
2 & 36 & 100 & 60 & 0 & 49 & 58 & 27 & 12 & 35 & 68 & 46 & 41 & 88 & 93 & 100 & 50 & 32 & 38 & 50 & 12 & 15 & 67 \\
3 & 41 & 100 & 100 & 11 & 29 & 44 & 29 & 10 & 9 & 100 & 77 & 33 & 100 & 37 & 100 & 24 & 31 & 47 & 58 & 100 & 50 & 75 \\
4 & 18 & 100 & 67 & 44 & 27 & 18 & 28 & 6 & 13 & 88 & 58 & 23 & 100 & 13 & 100 & 17 & 44 & 46 & 50 & 100 & 23 & 100 \\
5 & 100 & 100 & 100 & 18 & 33 & 31 & 26 & 8 & 29 & 52 & 60 & 36 & 80 & 61 & 100 & 32 & 39 & 30 & 44 & 0 & 0 & 78 \\
6 & 51 & 74 & 100 & 9 & 23 & 52 & 19 & 9 & 12 & 83 & 85 & 41 & 100 & 28 & 100 & 28 & 34 & 53 & 100 & 0 & 0 & 83 \\
\hline
\end{tabular}

Fonte: Elaborada pelos autores

É possível observar que os recursos passam a ter uma ocupação melhor em comparação ao Cenário 1, com alguns recursos atingindo 100\% de ocupação. Conforme já observado, estes recursos, com ocupação de 100\%, são gargalos que podem ser avaliados como candidatos a terem sua capacidade expandida. Por outro lado, é possível observar, novamente, grande ociosidade do recurso 8, que agora apresenta uma ocupação máxima de 12\%, ligeiramente superior ao valor máximo observado no cenário anterior.

Para o Cenário 3, no qual a demanda não tem restrição de quantidades mínimas ou máximas, o modelo apresentou a margem de contribuição total de $\mathrm{R} \$$ 409.840,00 que representa um ganho de aproximadamente 120\% em relação ao plano de produção realizado. Apesar de ser um cenário muito improvável de acontecer, ele tem uma função de análise gerencial, permitindo identificar, por exemplo, quais os produtos que trazem um maior retorno financeiro $e$, consequentemente, deveriam receber uma atenção diferenciada pelo setor comercial da empresa, com ações específicas de venda que aumentassem a sua participação no mercado. Ainda, a avaliação deste cenário permite identificar os gargalos de produção, fornecendo aos gestores informações relevantes para decisões quanto a investimentos futuros.

A Tabela 4 apresenta o percentual de ocupação de cada recurso em cada um dos períodos do horizonte de planejamento para o Cenário 3. 
Tabela 4 - Ocupação dos recursos para o Cenário 3 (\%)

\begin{tabular}{crrrrrrrrrrrrrrrrrrrrrr}
\hline Mês & $\mathbf{1}$ & $\mathbf{2}$ & $\mathbf{3}$ & $\mathbf{4}$ & $\mathbf{5}$ & $\mathbf{6}$ & $\mathbf{7}$ & $\mathbf{8}$ & $\mathbf{9}$ & $\mathbf{1 0}$ & $\mathbf{1 1}$ & $\mathbf{1 2}$ & $\mathbf{1 3}$ & $\mathbf{1 4}$ & $\mathbf{1 5}$ & $\mathbf{1 6}$ & $\mathbf{1 7}$ & $\mathbf{1 8}$ & $\mathbf{1 9}$ & $\mathbf{2 0}$ & $\mathbf{2 1}$ & $\mathbf{2 2}$ \\
\hline 1 & 14 & 60 & 2 & 0 & 12 & 4 & 30 & 3 & 18 & 35 & 11 & 10 & 43 & 1 & 25 & 2 & 11 & 8 & 33 & 100 & 30 & 27 \\
2 & 33 & 100 & 49 & 0 & 51 & 46 & 24 & 10 & 30 & 68 & 37 & 34 & 87 & 72 & 89 & 38 & 27 & 37 & 45 & 7 & 9 & 60 \\
3 & 37 & 100 & 99 & 11 & 28 & 43 & 28 & 10 & 10 & 100 & 71 & 32 & 100 & 38 & 100 & 26 & 30 & 46 & 56 & 100 & 50 & 73 \\
4 & 18 & 100 & 61 & 40 & 27 & 19 & 26 & 6 & 13 & 90 & 53 & 23 & 100 & 17 & 100 & 21 & 42 & 46 & 49 & 100 & 23 & 95 \\
5 & 100 & 100 & 100 & 18 & 33 & 36 & 19 & 8 & 26 & 53 & 61 & 35 & 78 & 77 & 100 & 40 & 38 & 28 & 43 & 0 & 0 & 75 \\
6 & 44 & 72 & 100 & 9 & 23 & 46 & 23 & 9 & 11 & 83 & 84 & 40 & 100 & 23 & 100 & 24 & 34 & 55 & 98 & 0 & 0 & 83 \\
\hline
\end{tabular}

Fonte: Elaborada pelos autores

É possível observar que alguns recursos permanecem com grande ociosidade; com destaque, mais uma vez, para o recurso 8 que apresenta uma ocupação máxima de $10 \%$.

\section{CONCLUSÕES}

A otimização do planejamento agregado da produção com uso da pesquisa operacional como ferramenta matemática mostrou-se um instrumento útil por proporcionar uma solução de natureza quantitativa para o problema. Com base na construção de cenários a partir de alterações nas restrições e variáveis especificadas, o estudo apresentou a solução ótima de cada cenário para atender a uma demanda prevista, destacando a margem de contribuição e o percentual de ocupação de cada recurso em cada um dos períodos do horizonte de planejamento. Essa última informação permitiu observar quais recursos passam a ter uma ocupação melhor, na comparação entre cenários. A possibilidade de avaliar quantitativamente o uso de cada recurso, permite apoiar os gestores na tomada de decisão relativa à expansão da capacidade de recursos sobrecarregados, bem como na definição de políticas adequadas para uso dos recursos ociosos.

Um aspecto do trabalho a ser destacado, que certifica as informações apresentadas, foi a rigorosa e variada coleta de dados realizada para o desenvolvimento do modelo matemático. Buscou-se o maior número de informações possível sobre o processo para que fossem apresentados os resultados mais próximos possíveis da realidade vivenciada pela empresa. Reuniu-se todas as 
informações do sistema ERP da empresa por meio de relatórios setoriais. Alguns dados tiveram que ser analisados e ajustados após se dar início ao estudo e entendimento do problema, devido às características dos processos e às necessidades para a formulação do modelo.

A simulação de novos cenários de forma rápida, proporcionada pelo modelo de otimização baseado em programação linear, possibilita aos gestores um maior conhecimento das características produtivas, como identificar as restrições do sistema, ou mesmo exibir ganhos que poderiam ser obtidos por meio de investimentos na ampliação da capacidade da produção. Informações que aumentam a assertividade de suas decisões. Além disso, a avaliação de diferentes cenários e seus resultados financeiros podem ser utilizados como justificativa para a aprovação de futuros investimentos junto ao alto executivo.

A geração de cenários também contribuiu para demonstrar com maior clareza a proposta prática da ferramenta de otimização. A avaliação das diferentes situações práticas, possivelmente vivenciadas na empresa, puderam ser rapidamente construídas através da alteração de alguns poucos parâmetros que foram estipulados para se diferenciar do cenário básico principal. O comparativo entre estes resultados evidencia o potencial de uma ferramenta desta natureza para o gerenciamento do planejamento da produção, possibilitando a automatização e o aprimoramento da estratégia de definição do mix ideal de produção.

Pode se concluir, portanto, que os resultados justificam a implantação de ferramentas baseadas na programação linear para o planejamento agregado da produção da empresa. Por meio da criação da base de dados do problema e da modelagem matemática, obtém-se o agrupamento de informações importantes do processo produtivo, bem como uma maior confiabilidade na escolha dos cenários de planejamento e tomadas de decisão.

\section{REFERÊNCIAS}

ANDRADE, E. L. Introdução à pesquisa operacional: métodos e modelos para análise de decisão. 5. ed. Rio de Janeiro: LTC, 2015. 636 p. 
ARENALES, M., et al. Pesquisa Operacional. 2. ed. Rio de Janeiro: Elsevier: ABEPRO, 2015. 744 p.

AZUMA, R. M. Otimização multiobjetivo em problema de estoque e roteamento gerenciados pelo fornecedor. 2011. $121 \mathrm{f}$. Dissertação (Mestrado em Engenharia de Computação) - Programa de Pós-Graduação em Engenharia Elétrica, Universidade Estadual de Campinas, Campinas, 2011.

BALLOU, R. H. Gerenciamento da cadeia de suprimentos/logística empresarial. 5. ed. Porto Alegre: Bookman, 2006. 616 p.

BARCELOS, B. O.; EVANGELISTA, M. L. S.; SEGATTO, S. S.. A importância e a aplicação da pesquisa operacional nos cursos de graduação em administração. RACE - Revista de Administração, Contabilidade e Economia da Editora Unoesc, v. 11, n. 2, p. 381-405. 2012.

CASSEL, L. G.; VACCARO, G. L. R.. A aplicação de simulação-otimização para definição do mix ótimo de produção de uma indústria metal-mecânica. In: ENCONTRO NACIONAL DE ENGENHARIA DE PRODUÇÃO, XXVII ENEGEP, 2002, Foz do Iguaçu. Anais... Foz do Iguaçu: ABEPRO, 2007.

CAVALCANTE, C. C. B.. Escalonamento com restrição de mão-de-obra: heurísticas combinatórias e limitantes inferiores. 1998. 136 f. Dissertação (Mestrado em Ciência da Computação) - Programa de Pós-Graduação em Ciência da Computação, Universidade Estadual de Campinas, Campinas, 1998.

CHERAGHALIKHANI A.; KHOSHALHAN, F.; MOKHTARI, H.. Aggregate production planning: A literature review and future research directions. International Journal of Industrial Engineering Computations, v. 10, p. 309-330, 2019.

http://dx.doi.org/10.5267/j.ijiec.2018.6.002

CHOPRA, S.; MEINDL, P. Supply chain management: strategy, planning, and operation. 2. ed. Boston, EUA: Pearson Education, 2004. 567 p.

CORRAR, L. J.; THEÓPHILO, C. R.. Pesquisa operacional: para decisão em contabilidade e administração - Contabilometria. 2. ed. São Paulo: Editora Atlas, 2004. 496 p.

DONATO, F. A. S. Otimização do mix de produtos e clientes em um planejamento agregado de produção - estudo de caso Embraco. 2008. 116 f. Dissertação (Mestrado em Engenharia de Produção) - Programa de Pós-Graduação em Engenharia de Produção, Universidade Federal de Santa Catarina, Florianópolis, 2008.

DOS REIS, S. G.; MARTINS, E. Planejamento do balanço bancário: desenvolvimento de um modelo matemático de otimização do retorno econômico ajustado ao risco. Revista Contabilidade e Finanças, São Paulo, v. 12, n. 26, p. 58-80, 2001. http://dx.doi.org/10.1590/S1519-70772001000200004

KRAJEWSKI, L. J.; RITZMAN, L. P.; MALHOTRA, M. K. Administração de produção e operações. 8. ed. São Paulo: Pearson, 2009. 615 p. 
LACHTERMACHER, G. Pesquisa operacional na tomada de decisões. 1. ed. Rio de Janeiro: Campus, 2002. 321 p.

LARA, V. R. Desenvolvimento de plano agregado de produção para um sistema agroindustrial. 2003. 146 f. Dissertação (Mestrado em Engenharia de Produção) Programa de Pós-Graduação em Engenharia de Produção, Universidade Federal do Rio Grande do Sul, Porto Alegre, 2003.

MONKS, J. G. Administração da produção. 1. ed. São Paulo: McGraw-Hill, 1987. 516 p.

MOREIRA, D. A. Administração da produção e operações. 2. ed. São Paulo: Pioneira, 1996. 619 p.

NAM, S-J; LOGENDRAN, R.. Aggregate production planning - A survey of models and methodologies. European Journal of Operational Research, v. 61, n. 3, p. 255-272, 1992. https://doi.org/10.1016/0377-2217(92)90356-E

ONWUBOLU, G. C. Emerging optimization techniques in production planning and control. 1. ed. Londres: Imperial College Press, 2002. 632 p. https://doi.org/10.1142/p228

OLIVEIRA, F. F.; SILVEIRA, R. R.; FERREIRA, R. J. P.. Pesquisa operacional como apoio na tomada de decisões em gestão da produção. In: SIMPÓSIO DE ENGENHARIA DE PRODUÇÃO, XVII SIMPEP, 2010, Bauru. Anais... Bauru: UNESP, 2010.

OLIVEIRA, L. H. S. Planejamento agregado da produção: um estudo de caso na indústria têxtil. 2011. Trabalho de Conclusão de Curso (Graduação em Engenharia de Produção) Departamento de Engenharia de Produção, Escola Politécnica, Universidade de São Paulo, São Paulo, 2011.

ROCHA, G. V. et al.. Otimização da programação da produção em manufatura calçadista. In: ENCONTRO NACIONAL DE ENGENHARIA DE PRODUÇ̃̃O, XXXII ENEGEP, 2012, Bento Gonçalves. Anais... Bento Gonçalves: ABEPRO, 2012.

RODRIGUES, D. S. S.; COSTA, H. G.; CARMO, L. F. R. R. S. Métodos de auxílio multicritério à decisão aplicados a problemas de PCP: mapeamento da produção em periódicos publicados no Brasil, Gestão \& Produção, São Carlos, v. 20, n.1, p. 134-146, 2013. http://dx.doi.org/10.1590/S0104-530X2013000100010

SILVA FILHO, O. S.; CEZARINO, W.; RATTO, J.. Planejamento agregado da produção: modelagem e solução via planilha Excel \& Solver. Revista Produção Online, v. 9, n. 3, p. 572-599, 2009. http://dx.doi.org/10.14488/1676-1901.v9i3.173

SILVEIRA, G. M. et al. Aplicação da programação linear para otimização de cortes de eixos na indústria automotiva. In: ENCONTRO NACIONAL DE ENGENHARIA DE PRODUÇÃO, XXXIII ENEGEP, 2013, Salvador. Anais... Salvador: ABEPRO, 2013.

SLACK, N. et al. Administração da produção. 1. ed. São Paulo: Atlas, 1997. 726 p.

TANCREDI, T. P. Otimização multidisciplinar distribuída aplicada a projetos de engenharia. 2009. 190 f. Tese (Doutorado em Engenharia) - Escola Politécnica, 
Universidade de São Paulo, São Paulo, 2009. http://dx.doi.org/10.11606/T.3.2009.tde20082009-114336

TOMAS, R. N.; SATO, L.; ALCANTARA, R. L. C. Planejamento de vendas e operações (S\&OP) no segmento de bens de consumo: uma análise envolvendo o estágio de maturidade do processo. Revista de Administração da UNIMEP, Piracicaba, v. 10, n. 3, 2012. http://dx.doi.org/10.15600/1679-5350/rau.v10n3p1-25

TUBINO, D. F. Planejamento e Controle da Produção. 2. ed. São Paulo: Atlas, 2000. 220 p.

VOLLMANN, T. E.; BERRY, W. L.; WHYBARK, D. C. Manufacturing planning and control systems. 4. ed. Boston, EUA: Irwin McGraw-Hill, 1997. 864 p.

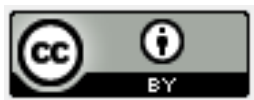

Artigo recebido em: 24/09/2017 e aceito para publicação em: 12/02/2019

DOI: http://dx.doi.org/10.14488/1676-1901.v19i1.3013 